\title{
Multifunctional hosts of Zinc sulfide coated carbon nanotubes for lithium sulfur batteries
}

\author{
Honghong $\mathrm{Liu}^{1,2} \cdot$ Tiju Thomas $^{3} \cdot{\text { Rongrong } \mathrm{Li}^{1,2} \cdot \text { Hangjia Shen }^{1,2} \text { (D) Jiacheng Wang }}^{1,2,4} \cdot$ Minghui Yang ${ }^{1,2}$
}

Received: 31 March 2020 / Accepted: 27 May 2020 / Published online: 2 June 2020

(c) Springer Nature Switzerland AG 2020

\begin{abstract}
Lithium sulfur (Li-S) batteries are next general energy storage systems due to their high thereotical energy density, low cost and environmental friendly. Herein, we develop a composite polysulfide mediator based on carbon nanotubes enwrapped by zinc sulfide (CNTs@ZnS) nanoparticles as multifunctional host materials for sulfur cathode. The ZnS nanoparticles is uniformed coating on the outside layer of functionalized carbon nanotubes. The unique structure of CNTs@ $\mathrm{ZnS}$ ensure the chemical adsorption on $\mathrm{ZnS}$ and fast electron transfer from $\mathrm{ZnS}$ to carbonnanotubes. The presence of $\mathrm{ZnS}$ efficiently alleviates the shuttle effect of soluble lithium polysulfide intermediates and hence offers a mean to further enhancing the reaction activity via sulfophilic property of $\mathrm{ZnS}$ and catalytically accelerate the conversion of polysulfides to insoluable $\mathrm{Li}_{2} \mathrm{~S}$. The high conductivity of carbon nanotubes results in rapid electron transfer. The CNTs@ZnS cathode offers an excellent specific capacity of $\sim 1003 \mathrm{mAh} \mathrm{g}^{-1}$ after 100 cycles at $0.2 \mathrm{C}$. Furthermore significant cycling performance at $0.2 \mathrm{C}$ with $3.1 \mathrm{mg} \mathrm{cm}^{-2}$ sulfur loading for 500 cycles is observed with $0.05 \%$ capacitance decay per cycle. The results provide insights to develop high-performance Li-S batteries based on multifunctional host materials.
\end{abstract}

Keywords Zinc sulfide · Carbon nanotubes · Lithium sulfur batteries · Chemical adsorption · Enhanced reaction activities

\section{Introduction}

With the industrial and economic growth, energy plays a critical role in present and future. The needs of energy are evidently growing and there is an evident need for reorganization of the economy around renewable energy sources. This in turn implies the need for large scale rechargeable energy storage devices [1-3]. In order to improve the efficiency of electric power utilization, stable and sustainable energy storage systems with low cost and eco-friendly are essential [4, 5]. Lithium sulfur $(\mathrm{Li}-\mathrm{S})$ batteries are emerging as a promising new generation of energy storage devices for electric vehicles and portable energy devices [6-8]. It is known that the $\mathrm{Li}-\mathrm{S}$ batteries possess high theoretical specific capacity of $1675 \mathrm{mAh} \mathrm{g}^{-1}$ and energy density of $2600 \mathrm{Wh} \mathrm{kg}^{-1}$, which is five times higher than that of commercially available lithium ion batteries [8-10]. Meanwhile, the low cost and abundance of sulfur make it possible for large scale energy storage $[11,12]$. However, there are

Electronic supplementary material The online version of this article (https://doi.org/10.1007/s42452-020-2964-0) contains supplementary material, which is available to authorized users.

Hangjia Shen, shenhj@nimte.ac.cn; $\bowtie$ Jiacheng Wang, jiacheng.wang@mail.sic.ac.cn; $\bowtie$ Minghui Yang, myang@nimte.ac.cn | 'Solid State functional Materials Research Laboratory, Ningbo Institute of Materials Technology and Engineering, Chinese Academy of Sciences, Ningbo 315201, China. ${ }^{2}$ Center of Materials Science and Optoelectronics Engineering, University of Chinese Academy of Sciences, Beijing 100049, China. ${ }^{3}$ Department of Metallurgical and Materials Engineering, Indian Institute of Technology Madras, Adyar, Chennai, Tamil Nadu 600036, India. ${ }^{4}$ The State Key Laboratory of High Performance Ceramics and Superfine Microstructure, Shanghai Institute of Ceramics, Chinese Academy of Sciences, 1295 Dingxi Road, Shanghai 200050, China. 
still significant obstacles for the practical application of Li-S batteries [13-15].

The major concern revolves the low utilization of sulfur and the slow kinetics of the associated electrochemical redox reactions because the electrically insulation of sulfur and $\mathrm{Li}_{2} \mathrm{~S}$ limits electrons and lithium ions transportation in the electrode $[16,17]$. Meanwhile, the intermediate polysulfides $\left(\mathrm{Li}_{2} \mathrm{~S}_{x}, 2<x<8\right)$ are soluble in the organic electrolytes and they shuttle between anode and cathode. The dissolved polysulfides directly react with lithium ions and electrons on the lithium anode surface (shuttle effect); which results in low Coulombic efficiency, fast capacity decay and poor cycle stability $[7,18]$. Finally, the large volume expansion of the sulfur particles leads to the breakage of cathode's structure and rapid capacity fading [19].

Currently, extensive research efforts have been devoted to enhance the electrochemical performance of cathode materials $[10,20]$. Various carbon-based materials including carbon nanofiber [21], carbon nanotube and graphene have been designed as effective cathode hosts for sulfur loading. These carbon based materials can restrain the shuttle effect by physical confinement and improve the conductivity of the cathodes [22]. Unfortunately, the nonpolar carbon-based materials used as cathodes, exhibit weak interaction with polar lithium polysulfide intermediates (LiPSs). This in fact results in the degradation of cycling stability and rate capability. On the other hand, polar inorganic metal-based materials can efficiently prevent the loss of active sulfur materials and hence chemically anchor LiPSs for advanced Li-S batteries. Recently, metal oxides such as $\mathrm{MoO}_{2}$ [23], $\mathrm{TiO}_{2}$ [24, 25], $\mathrm{SnO}_{2}$ [26] and $\mathrm{MnO}_{2}[27,28]$ have been explored as chemical trappers to bind LiPSs through polar chemical interaction in the cathodes. However, due to the poor conductivity of metal oxides, the fabricated $\mathrm{Li}-\mathrm{S}$ batteries exhibit poor rate performance and low sulfur utilization. Furthermore, the sluggish reaction kinetics make the LiPSs assemble on the host surface and cause the adsorption of LiPSs decreased. For above reasons, an ideal sulfur host material should have high conductivity to insure the sufficient utilization of sulfur and should also have strong electrocatalytic activity to promote the conversion of LiPSs. To achieve this, metal sulfides (e.g. $\mathrm{Co}_{9} \mathrm{~S}_{8}, \mathrm{MoS}_{2}, \mathrm{WS}_{2}$, $\mathrm{SnS}_{2}$, etc.) have been investigated as cathode materials for $\mathrm{Li}-\mathrm{S}$ batteries. This is because of the strong sulfiphilic property of metal sulfides to sulfur-containing species [29, 30]. Furthermore, the metal sulfides can act as an activation catalyst to promote the oxidation of $\mathrm{Li}_{2} \mathrm{~S}$, as indicated by Cui et al. [8]. As typical metal sulfides, zinc sulfide (ZnS) possesses the strong sulfiphilic property to LiPSs. Moreover, the polar feature of $\mathrm{ZnS}$ is also beneficial to adsorption and catalytic conversion of polar LiPSs.
Herein, zinc sulfide ( $\mathrm{ZnS}$ ) coated carbon nanotubes (CNTs@ZnS) are synthesized as sulfur host with strong chemical adsorption and electrocatalytic activity towards LiPSs conversion for high performance $\mathrm{Li}-\mathrm{S}$ batteries. The CNTs@ZnS composite is prepared using simple self-assembly of ZnS and CNTs. It is then thermally treated under argon. The product is composed of zinc sulfide nanoparticles, which assemble on carbon nanotubes. The core of carbon nanotubes can enhance the conductivity for electrons/ions transfer and provide physical entrapment of LiPSs. The ZnS acts as a sulfiphilic host that promotes the LiPSs redox process and improves the chemical adsorption to effectively trap the polysulfides. With the advantage of the synergetic effect between CNTs and ZnS, the sulfur loaded CNTs@ZnS cathode has a large capacity of 1003 $\mathrm{mAh} \mathrm{g}^{-1}$ after 100 cycles at $0.2 \mathrm{C}$. And the long cycle life with low capacity fading of $0.05 \%$ per cycle over 500 cycles at $0.2 \mathrm{C}$ with $3.1 \mathrm{mg} \mathrm{cm}^{-2}$ sulfur loading is observed in the Li-S batteries.

\section{Experimental}

\subsection{Materials synthesis}

Synthesis of carbon nanotubes (CNTs). A facile wet chemical method was employed to synthesize the carbon nanotubes (CNTs). In a typical experiment, $98 \mathrm{mg}(0.3 \mathrm{mmol})$ of methyl orange powders ( $98 \%$, Tokoyo Chemical) were dissolved in $60 \mathrm{~mL}$ deionized water under continuously stirring. Then $0.49 \mathrm{~g} \mathrm{(} 3 \mathrm{mmol}$ ) of anhydrous $\mathrm{FeCl}_{3}$ powder was added into the above solution and a flocculent precipitate was formed immediately. $210 \mu \mathrm{L}$ of pyrrole monomer (99\%, Admas Reagent) was mixed in the solution and kept at $25^{\circ} \mathrm{C}$ for $24 \mathrm{~h}$. The black polypyrrole nanotubes were collected and washed with deionized water and ethanol several times until the filtrate turned colorless and neutral. The produced PPy-NTs were finally freeze-dried for 24 h. Then, $100 \mathrm{mg}$ of the as-prepared PPy-NTs was placed in a quartz tube furnace and then heated under Ar environment from room temperature to $800^{\circ} \mathrm{C}$ at a rate of 10 ${ }^{\circ} \mathrm{C} \mathrm{min}^{-1}$ and kept for $3 \mathrm{~h}$. After the furnace was cooled down to room temperature, the black CNTs powder was obtained.

Synthesis of CNTs@ZnS. The resultant CNTs@ZnS was obtained by hydrothermal method. $20 \mathrm{mg}$ of CNTs was dispersed in $60 \mathrm{~mL}$ of ethylene glycol under stirring. Then, $0.46 \mathrm{~g}(1.3 \mathrm{mmol})$ of cetyltrimethylammonium bromide (CTAB, Aladdin) powder, $0.37 \mathrm{~g}(1.2 \mathrm{mmol})$ of $\mathrm{Zn}\left(\mathrm{NO}_{3}\right)_{2} \cdot 6 \mathrm{H}_{2} \mathrm{O}$ and $0.1 \mathrm{~g}(1.3 \mathrm{mmol})$ of thioacetamide were dissolved in the above solution. The solution was then transferred to a $100 \mathrm{~mL}$ Teflon-lined autoclave and heated to $160^{\circ} \mathrm{C}$ for $12 \mathrm{~h}$. After cooling to room 
temperature, the gray product was collected using centrifugation and further washed several times using ethanol and deionized water, and then dried at $60^{\circ} \mathrm{C}$ for $18 \mathrm{~h}$. Finally, the CNTs@ZnS was obtained by annealing the gray precursor under $\mathrm{Ar}$ atmosphere at $500^{\circ} \mathrm{C}$ for $2 \mathrm{~h}$.

Synthesis of S/CNTs@ZnS. A mixture of CNTs@ZnS and sulfur $(70 \%$, weigh ratio) was sealed in a glass bottle, and heated at $155^{\circ} \mathrm{C}$ for $24 \mathrm{~h}$ in an oven. The S/CNTs was prepared using the same method without $\mathrm{ZnS}$ coating.

\subsection{Structural characterizations}

The phases and chemical compositions of all the products were analyzed by X-ray diffraction (XRD, D/max-RB, Rigaku with Cu ka radiation). The morphology of the samples was performed using a scanning electron microscopy (SEM, JSM-7800F), transmission electron microscopy (F200X-G2) and field emission scanning transmission electron microscope (STEM). The sulfur content was measured under a nitrogen atmosphere through thermal gravimetric analysis (TGA). $\mathrm{N}_{2}$ adsorption/desorption isothermals were characterized by a gas adsorption analyzer (ASAP-2020). The Brunauer-Emmett-Teller (BET) method was used to calculate the specific surface areas and the Quenched Solid Density Function Theory (QSDFT) was used to calculate the pore-size distribution. UV-visible spectra were obtained using a Microsolar 300 UV-Vis absorption spectroscopy. The chemical composition were measured by XPS measurements (ESCALAB250 X-ray photoelectron spectrometer).

\subsection{Polysulfide adsorption test}

A polysulfide electrolyte was prepared using excess sulfur chemically reacting with lithium metal in electrolyte to form saturated solution of polysulfides. Subsequently, 10 mg of CNTs@ZnS and CNTs were respectively immersed into $1 \mathrm{~mL}$ of polysulfides saturated solution and electrolyte $(v / v=9: 1)$. After resting for $12 \mathrm{~h}$, the digital photos were taken to display the discoloration of the above samples and the adsorption precipitated product of CNTs@ZnSLiPSs was studied using X-ray photoelectron spectroscopy.

\subsection{Electrochemical measurements}

The working cathodes were prepared by mixing $10 \%$ carbon black (Super P), 10\% poly(vinylidenefluoride) binder (PVDF) and $80 \%$ active materials in $\mathrm{N}$-methyl-pyrrolidone (NMP), followed by casting the slurry onto carbon paper and drying at $60^{\circ} \mathrm{C}$ for $12 \mathrm{~h}$ in a vacuum oven. Finally, the slurry cathode was cut into discs with a diameter of 12 $\mathrm{mm}$. The electrolyte/sulfur ratio was about $20 \mu \mathrm{L} \mathrm{mg}^{-1}$ for cathodes. The 2032-type coin cells were assembled using lithium foil as the anode and Celgard 2325 as membranes. The electrolyte used was a freshly prepared solution of lithium bis(trifluoromethanesulfonyl) imide. Electrochemical impedance spectroscopy (EIS) measurements and cyclic voltammetry $(\mathrm{CV})$ were performed on an electrochemical workstation (CHI660E, Shanghai, China). CV curves were performed with a scan rate of $0.1 \mathrm{mV} \mathrm{s}^{-1}$ between 1.8 and 3.0 V. Electrochemical Impedance Spectroscopy was tested in the frequency range between $0.01 \mathrm{~Hz}$ and $1000 \mathrm{kHz}$ at an open circuit voltage of each cell. And galvanostatic cycling was carried out from 1.8 to $2.8 \mathrm{~V}$ using a battery measurement system (Neware, China).

\subsection{Symmetrical cell assembly and electrochemical test}

The symmetrical cells are designed by sandwiching lithium bis(trifluoromethanesulfony)imide (LiTFSI) electrolyte containing 0.5 M LiPSs between two identical CNTs@ ZnS or CNTs electrodes as both the working and counter electrodes. The active materials and polyvinylidene difluoride (PVDF) binder (4:1, ratio by weight) were mixed in N-Methyl prrolidone (NMP). The electrode foil was punched to disks with a diameter of $12.0 \mathrm{~mm}$ and areal sulfur loading of $1-1.5 \mathrm{mg} \mathrm{cm}^{-2}$. The coin cells were assembled with two same disks as the cathode and anode, Celgard 2400 separator, and $40 \mu \mathrm{L}$ catholyte containing $0.5 \mathrm{M}$ LiTFSI and 0.5 M LiPSs in DME/DOL $(v / v=1 / 1)$. CV measurements were obtained from a symmetric cell using a scanning rate of $3 \mathrm{mV} \mathrm{s}^{-1}$ with potential window between -1.5 and $1.5 \mathrm{~V}$.

\section{Results and discussion}

Figure 1a shows a schematic illustration of the preparation procedures of the S/CNTs@ZnS composite. As observed in scanning electron microscope (SEM) image in Fig. 1b, the carbon nanotubes (CNTs) obtained by the direct carbonization of polypyrrole-NTs shows a length of 3-5 $\mu \mathrm{m}$ and a diameter of 100-150 nm with smooth surface. These CNTs are mixed with zinc nitrate and thioacetamide solution, followed hydrothermal treatment at $160^{\circ} \mathrm{C}$ for $12 \mathrm{~h}$. Subsequently calcining is carried out at $500^{\circ} \mathrm{C}$ for $2 \mathrm{~h}$ in argon gas to obtain ZnS nanoparticles coated on CNTs (CNTs@ZnS). As shown in Fig. 1c, the as-formed CNTs@ZnS maintains the nanotube morphology of CNTs with small ZnS nanoparticles coated on CNTs. The thickness of outer layer is 50-80 $\mathrm{nm}$ thickness, which can be clearly seen in TEM image (Fig. 2a). Meanwhile a certain mass of sulfur is homogeneously loaded on the CNTs@ZnS via physical deposition at $155^{\circ} \mathrm{C}$ for $12 \mathrm{~h}$ to fabricate the aimed S/CNTs@ZnS. After sulfur loading, the morphology of S/ 
Fig. 1 a Schematic illustration of the fabrication processes of S/CNTs@ZnS. b-d SEM images of $\mathbf{b}$ carbon nanotubes, $\mathbf{c}$ CNTs@ZnS and d S/CNTs@ZnS
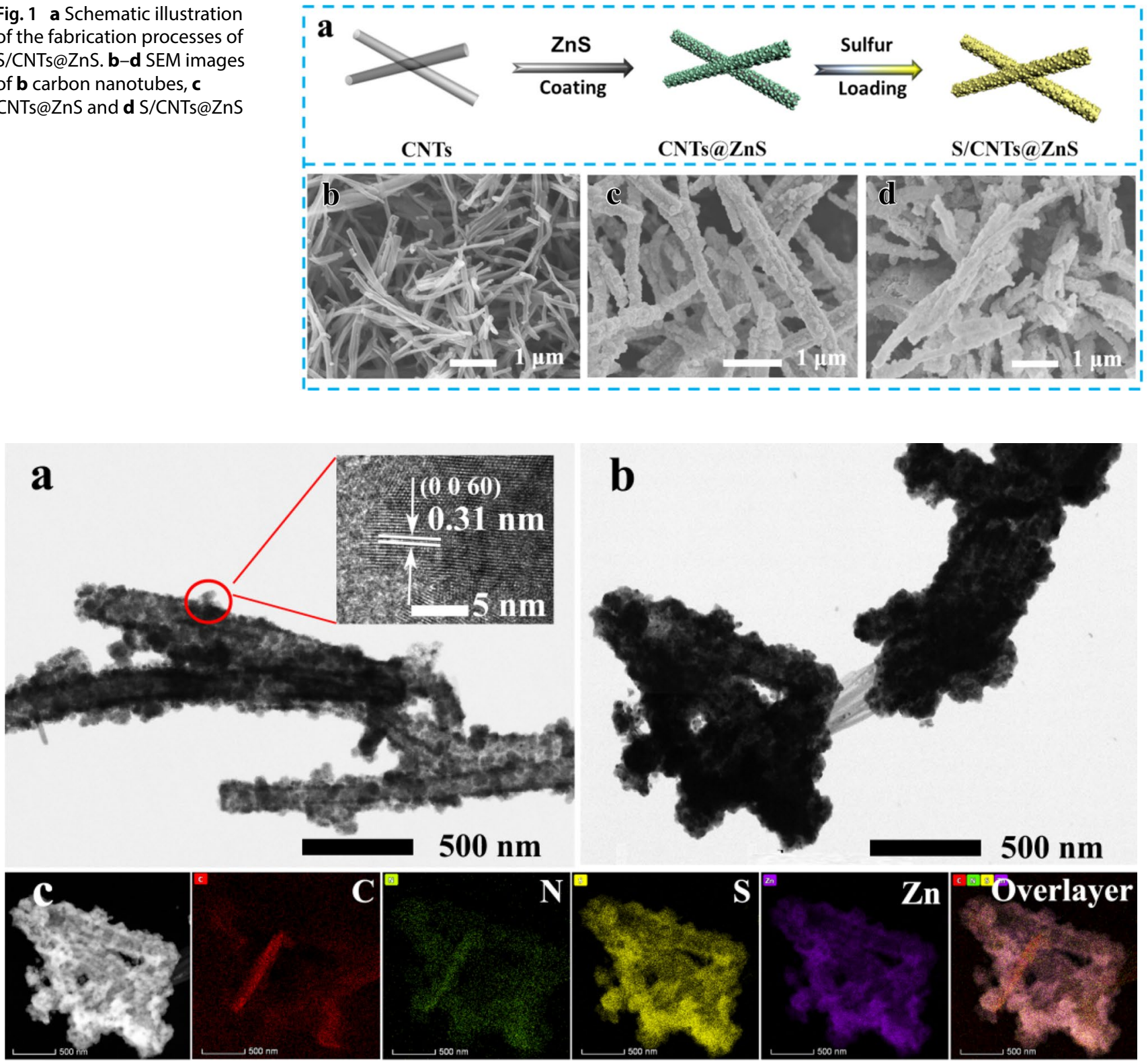

Fig. 2 a TEM image of CNTs@ZnS (inset: HRTEM image). b TEM image of S/CNTs@ZnS. c STEM image of S/CNTs@ZnS and corresponding element maps of $C, N, S$ and $Z n$. Scale bars, $500 \mathrm{~nm}$

CNTs@ZnS is shown in Fig. 1d. The sulfur is distributed homogenous within CNTs@ZnS. And the sulfur content of S/CNTs@ZnS is measured to be 70 wt \% (Figure S3). As confirmed by X-ray diffraction (XRD) pattern in Figure S1, only peaks attributable to $\mathrm{ZnS}$ (JCPDS 89-2173) are founded at $28.5^{\circ}, 47.5^{\circ}$ and $56.4^{\circ}$. For comparison, the ZnS nanospheres (Figure S2) obtained without CNTs get agglomerated. Correspondingly, in the XRD curve of S/CNTs@ZnS (Figure S1), orthorhombic sulfur (JCPDS 08-0247) related peaks are observed.

The $\mathrm{N}_{2}$ adsorption/desorption profiles of CNTs@ZnS and CNTs are presented in Figure S4. Compared with CNTs (64.0 $\left.\mathrm{m}^{2} \mathrm{~g}^{-1}\right)$, the low specific surface area of CNTs@ZnS $\left(41.4 \mathrm{~m}^{2}\right.$ $\mathrm{g}^{-1}$ ) is ascribed to the existence of $\mathrm{ZnS}$ on the surface. To furxther investigate the structure and the elemental distribution of synthesized samples, the transmission electron microscope (TEM) images are further analyzed. As shown in Fig. 2a, a tubular structure with a diameter of 150-200 $\mathrm{nm}$ is clearly observed. This is in consistent with the SEM analysis. ZnS nanoparticles are founded to be interconnected along with 1D porous carbon nanotubes. In the high resolution transmission electron microscope (HRTEM) image (Fig. 2a, inset), a lattice fringe with space of 0.31 $\mathrm{nm}$ can be clearly observed, and is indexed to the $\left(\begin{array}{ll}0 & 060\end{array}\right)$ 
plane of trigonal phase $\mathrm{ZnS}$. The corresponding element mappings of CNTs@ZnS is shown in Figure S5. After loading sulfur, the TEM image of S/CNTs@ZnS in Fig. 2b reveals that the overall morphology is still retained. The HAADF image and the corresponding energy-dispersive $X$-ray spectroscopy (EDS) mappings of S/CNTs@ZnS is depicted in Fig. 2c. It suggests that the sulfur is either in $\mathrm{ZnS}$ or in the form of the sulfur deposited in the post loading step. Furthermore it is clear that the sulfur content is uniform on the CNTs.

In order to further probe the strong polysulfide capture capability of CNTs@ZnS, the visual test and ultraviolet/visible absorption spectra are carried out in Fig. 3a. This is done by dispersing $10 \mathrm{mg}$ of CNTs@ZnS or CNTs powder in the polysulfide-saturated solution. Without the chemical capture capability between LiPSs and the polar cathode material, a large amount of intermediate polysulfide can easily diffuse away from the carbon cathode, resulting in serious shuttle effect and deposition of insoluable $\mathrm{Li}_{2} \mathrm{~S}_{x}$ $(x \leq 2)$. The adsorption ability of CNTs@ZnS composite can be shown by the color change of the typical polysulfides solution. As shown in Fig. 3a (inset), after $12 \mathrm{~h}$ aging, the polysulfides are effectively adsorbed by the immersing CNTs@ZnS; this correlates with change in color of corresponding solution (becomes nearly transparent). In contrast, the color of the polysulfides solution with CNTs shows no obvious color fading. And it can be observed that there are two obvious absorption peaks of pristine LiPSs solution in the range at around $450 \mathrm{~nm}$ and $330 \mathrm{~nm}$. The two peaks can be attributed to the absorbance peak of $\mathrm{Li}_{2} \mathrm{~S}_{6} / \mathrm{Li}_{2} \mathrm{~S}_{4}$ and $\mathrm{Li}_{2} \mathrm{~S}_{8} / \mathrm{Li}_{2} \mathrm{~S}_{6}$ [31]. The UV-Vis spectrum shows the absorbance for the solution of CNTs@ZnS after adsorption is nearly absents at $420 \mathrm{~nm}$, confirming that the $\mathrm{Li}_{2} \mathrm{~S}_{6} /$ $\mathrm{Li}_{2} \mathrm{~S}_{4}$ species are absorbed by the CNTs@ZnS composite. The result indicates the strong chemical adsorption ability ofCNTs@ZnS.

The X-ray photoelectron spectrum of CNTs@ZnS and CNTs@ZnS-LiPSs are further performed to provide proof for the strong chemical adsorption of LiPSs. The fine Li 1 s spectrum of CNTs@ZnS (Fig. 3b) corresponds to a peak at $55.9 \mathrm{eV}$ in addition to $\mathrm{Li}-\mathrm{S}$ bond after adsorption of LiPSs. This in fact results in a $0.6 \mathrm{eV}$ slightly shift to lower binding energy compared to the pristine $\mathrm{Li}_{2} \mathrm{~S}_{6}(56.3 \mathrm{eV})$ as literature reported [8]. The shift in the peak indicates the fast electron transfer from $\mathrm{ZnS}$ to lithium ion in the polysulfide solution. Also as shown in Fig. $3 c, Z n 2 p_{3 / 2}$ peak of the CNTs@ZnS after loading of LiPSs is located at 1021.6 $\mathrm{eV}$; which shifts to lower binding energy compared to that of CNTs@ZnS (1022.2 eV). It means that there exists a chemical reaction occurring between $\mathrm{ZnS}$ and LiPSs, which results in strong chemical bonds for LiPSs confinement. The Li $1 \mathrm{~s}$ and $\mathrm{Zn} 2 \mathrm{p}_{3 / 2}$ peaks indicate electron transfer
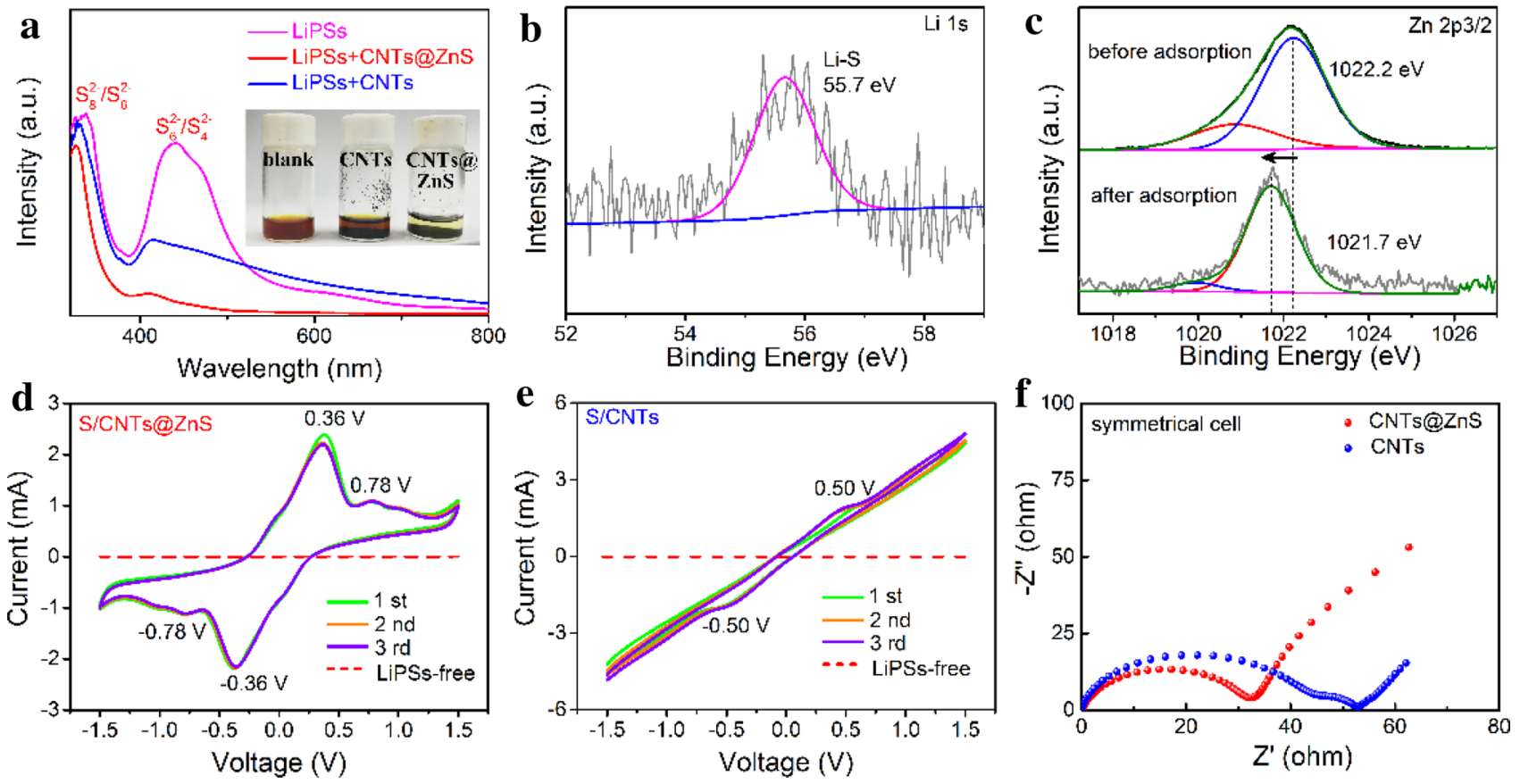

Fig. 3 a UV-Vis spectra of LiPSs solution and upper solution after adsorption of the CNTs@ZnS and CNTs. The inset is photo of LiPSs adsorption performance after addition of CNTS and CNTs@ZnS. b XPS spectrum of Li $1 \mathrm{~s}$ for CNTs@ZnS after adsorbing LiPSs. c XPS spectrum of Zn 2p3/2 for CNTs@ZnS before and after adsorption of LiPSs. CV curves of LiPSs and LiPSs-free symmetrical cell with d CNTs@ZnS and e CNTs working electrode with $1.2 \mathrm{mg} \mathrm{cm}^{-2}$ sulfur loading each disk at a scan rate of $3 \mathrm{mV} \mathrm{s}^{-1}$. $\mathbf{f}$ EIS of the LiPSs symmetrical cell with CNTs@ZnS and CNTs cathodes 
from LiPSs to the $\mathrm{Zn}$ atoms; which in turn. results in strong chemical adsorption.

To further probe the catalytic activity of CNTs@ZnS to LiPSs conversion in Li-S battery, symmetrical cells are designed for cyclic voltametric test in the voltage range -1.5 to $1.5 \mathrm{~V}$. The tests are done using an $3 \mathrm{mV} \mathrm{s}^{-1}$ scanning rate for LiPSs and LiPSs-free symmetrical cells [32]. As shown in Fig. 3d-e, the CV curve of the LiPSs-free one (red dotted lines) shows a minor contribution to the current. With the addition of LiPSs into the counter electrodes, the symmetrical cells show a much higher current intensity, which means that the chemical reactions of lithiation with CNTs@ZnS devote the current response. And four peaks are observed at $-0.78,-0.36,0.36$ and $0.78 \mathrm{~V}$ respectively. The LiPSs symmetrical cell with the CNTs@ZnS electrode shows facile polysulfide conversion and high reversibility in the first three cycles, which is much better than the LiPSs symmetrical cell with the CNTs (Fig. 3e). This clearly demonstrates the key role of $\mathrm{ZnS}$ in accelerating the electrochemical reactions with LiPSs. Meanwhile, the current density of CNTs@ZnS symmetrical cell is also higher than that of LiPSs symmetrical cell with ZnS electrode (Figure S6); this is a robust indicator of the role of carbon nanotubes in enhancing the conductivity. The electrochemical impedance spectroscopy (EIS) spectrum of the CNTs@ $\mathrm{ZnS}$ and CNTs electrodes symmetrical cell are presented in Fig. 3f. The charge transfer resistance (Rct) on the CNTs@ ZnS polysulfide interface is faster than that on the CNTspolysulfide interface. Considering that the symmetric cells are free of lithium foil, the faster charge transfer can be ascribed to the interfacial affinity between CNTs@ZnS and LiPSs and rapid charge transfer in the redox reaction of polysulfide conversion. This is also consistent with the CV results.

The electrochemical performance of S/CNTs@ZnS cathode is measured using the CR2032 cells with a lithium foil as the anode and DOL/DME (1:1, v/v) with $1 \mathrm{M}$ LiTFSI and $2 \% \mathrm{LiNO}_{3}$ as the electrolyte. The S/CNTs and S/ZnS electrodes with same sulfur loading $\left(1.2-1.3 \mathrm{mg} \mathrm{cm}^{-2}\right)$ as $\mathrm{S} /$ CNTs@ZnS is used as the experimental control. Figure 4a presents the CV curves of the S/CNTs@ZnS electrode for the first five cycles in the $1.8-3.0 \mathrm{~V}$ at a scan rate of $0.1 \mathrm{mV}$ $\mathrm{s}^{-1}$. The CV curves show two well-defined cathodic peaks at 2.3 and $2.03 \mathrm{~V}$; this is ascribed to the redox reaction of sulfur to long-chain lithium polysulfide $\mathrm{Li}_{2} \mathrm{~S}_{\mathrm{x}}(4 \leq \mathrm{x} \leq 8)$ and consecutive conversion of $\mathrm{Li}_{2} \mathrm{~S}_{\mathrm{x}}$ to short-chain sulfides $\left(\mathrm{Li}_{2} \mathrm{~S}_{2} / \mathrm{Li}_{2} \mathrm{~S}\right)$, respectively [33]. In the anodic sweep, the oxidation peaks at 2.34 and $2.41 \mathrm{~V}$ correspond to the oxidation of $\mathrm{Li}_{2} \mathrm{~S} / \mathrm{Li}_{2} \mathrm{~S}_{2}$ to long-chain polysulfides and ultimately to sulfur. For the initial five cycles, the peaks have no obvious shifts, indicating good electrochemical stability. Meanwhile compared with S/CNTs and S/ZnS (Figure S7), the S/CNTs@ZnS exhibits higher cathodic peaks potential, smaller anodic peaks and larger peaks currents. It suggests that the composite of CNTs@ZnS kinetically accelerates polysulfides redox conversion in some degree and is good for the fast transfer of electrons. Figure $4 \mathrm{~b}$ compares the discharge curves of the S/CNTs@ $\mathrm{ZnS}$ and S/CNTs electrodes at $0.5 \mathrm{C}\left(1 \mathrm{C}=1673 \mathrm{~mA} \mathrm{~h} \mathrm{~g}^{-1}\right)$. The S/CNTs@ZnS composite exhibits much higher initial
Fig. 4 Electrochemical performance of S/CNTs@ZnS and S/CNTs. a CV curve of Li-S battery based on the S/CNTs@ $\mathrm{ZnS}$ and S/CNTs cathode with above $1.2 \mathrm{mg} \mathrm{cm}^{-2}$ sulfur loading at the scanning rate of 0.1 $\mathrm{mV} \mathrm{s}$; b Typical voltage profile of S/CNTs@ZnS and S/CNTs at $0.5 \mathrm{C}$ and the corresponding discharge capacity in stage 1 and 2 for S/CNTs@ZnS and S/ CNTs are shown in inset image; c Electrochemical impedance spectra of S/CNTs@ZnS and $\mathrm{S} / \mathrm{CNTs}$ at the open circuit voltage (inset: the equivalent circuits); d Rate performance at different C-rates of S/CNTs@ ZnS and S/CNTs
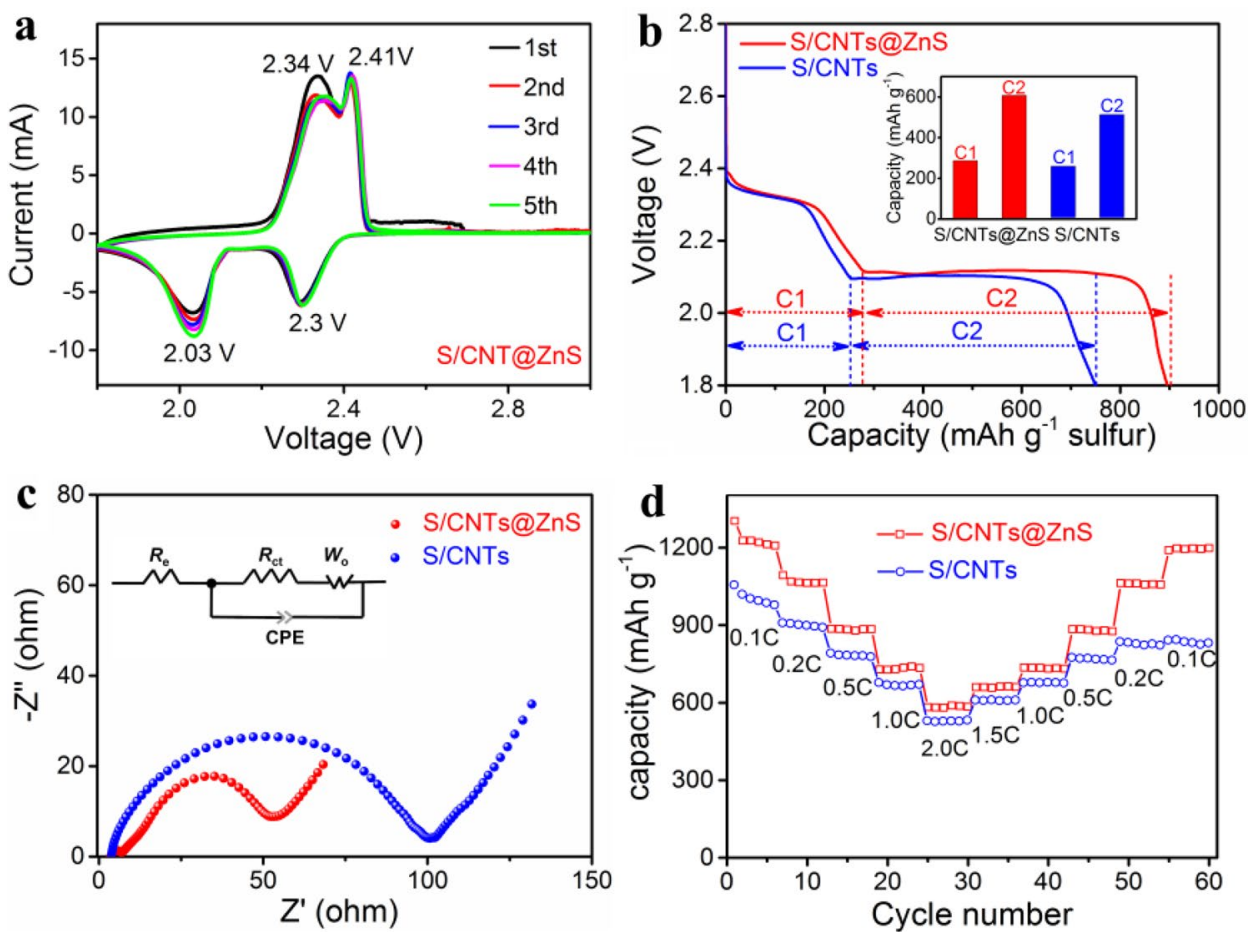
discharge capacities of $897 \mathrm{mAh} \mathrm{g}^{-1}$ in comparison with S/CNTs composite $\left(751 \mathrm{mAh} \mathrm{g}^{-1}\right)$, with initial Coulombic efficiency of $98.6 \%$. C1 and C2 are the discharge capacities of two typical plateaus corresponding to the conversion of sulfur to soluble polysulfides and further reduction to insoluble $\mathrm{Li}_{2} \mathrm{~S}$. Moreover, the capacity ratio $(\mathrm{C} 2 / \mathrm{C} 1)$ of $\mathrm{S} /$ CNTs@ZnS indicates improved polysulfide redox kinetics and higher sulfur utilization when compared with S/CNTs [34], which agrees with the results of CV curves of the symmetrical cells and lithium sulfur cell. The enhanced redox kinetics of S/CNTs@ZnS can be attributed to the synergetic effects of $\mathrm{ZnS}$ and CNTs. It provide higher electric conductivity for fast charge transfer of $\mathrm{Li}^{+} / \mathrm{e}^{-}$and also offers effective chemical adsorption of polysulfides for the conversion to $\mathrm{li}_{2} \mathrm{~S}_{2} / \mathrm{Li} \mathrm{i}_{2} \mathrm{~S}$ [35].

The EIS analysis and the corresponding equivalent circuit of the S/CNTs@ZnS,S/CNTs and S/ZnS electrodes after 10 cycles are displayed in Fig. $4 \mathrm{c}$ and Figure S8. As we all know, the start point of EIS spectra is the electrolyte resistance $\left(R_{e}\right)$ and the semicircle represents the charge transfer resistance $\left(R_{c t}\right)$ and relative capacitance (CPE). The sloped line in low frequency range is corresponds to the Warburg impedance (Wo), related to the diffusion of lithium ion in the electrode $[36,37]$. A smaller charge transfer resistance $(49.8 \Omega)$ on the S/CNTs@ZnS electrode when compared to S/CNTs $(97.0 \Omega)$ and S/ZnS (144.8 $\Omega$ ) indicates that the internal resistance of the Li-S battery system is reduced by the interaction of ZnS and CNTs. The results is consistent with $\mathrm{CV}$ results.

The lower resistance of charge transfer implies faster charge transfer rate due to the enhanced interfacial affinity and faster redox kinetics on the S/CNTs@ZnS electrode [23, 38]. Figure 4d shows the rate capability of the S/CNTs@ZnS and S/CNTs cathode electrodes at various rates from 0.1 to $2 \mathrm{C}$. With the current increase, the capacity decreases, and the S/CNTs@ZnS cathode has larger capacities and rate capability than that of S/CNTs cathode at all rate. Especially, when the current restore to $0.1 \mathrm{C}$ after 54 cycles, the capacity (1181 mA h g ${ }^{-1}$ ) of S/CNTs@ZnS cathode shows no abrupt degradation; this is much better than S/CNTs $\left(838 \mathrm{~mA} \mathrm{~h} \mathrm{~g}^{-1}\right)$, indicating high electrochemical reversibility.

The long term cycling performance of S/CNTs@ZnS is shown in Fig. 5. Figure 5a and Figure $S 9$ show the cycling performance and Coulombic efficiency of the S/CNTs@ $\mathrm{ZnS}$ and S/CNTs cathodes at $0.2 \mathrm{C}$ and $1 \mathrm{C}(1 \mathrm{C}=1672 \mathrm{mAh}$ $\mathrm{g}^{-1}$ ). It can be observed that the existence of $\mathrm{ZnS}$ nanoparticles alleviates the polysulfide shuttle effect with $1.51 \mathrm{mg} \mathrm{cm}^{-2}$ areal sulfur loading. As shown in Fig. $5 \mathrm{a}, \mathrm{S} /$ CNTs@ZnS cathode yields an initial capacity of 1069 mA $\mathrm{h} \mathrm{g}^{-1}$. Furthermore it retains a high reversible capacity of $1003 \mathrm{~mA} \mathrm{~h} \mathrm{~g}^{-1}$ after 100 cycles at $0.2 \mathrm{C}$ with Coulombic efficiency close to $100 \%$. In contrast, the initial capacity of S/CNTs is $905 \mathrm{~mA} \mathrm{~h} \mathrm{~g}^{-1}$ and after 100 cycles the capacity $\left(813 \mathrm{mAh} \mathrm{g}^{-1}\right)$, which is lower than S/CNTs@ZnS. S/ $\mathrm{ZnS}$ cathode were also prepared to assess the structure advantage of CNTs@ZnS. As shown in Figure S10, the S/ ZnS electrodes exhibit a low initial specific capacity of $1055 \mathrm{mAh} \mathrm{g}^{-1}$ at $0.2 \mathrm{C}$ and the capacity quickly drops to $717 \mathrm{mAh} \mathrm{g}^{-1}$ after 100 cycles, suggesting the low sulfur utilization than S/CNTs@ZnS. Meanwhile, the S/CNTs@ ZnS cathode exhibits slower capacity decay $(0.06 \%$ per cycle) than S/CNTs ( $0.1 \%$ per cycle) and S/ZnS (0.3\% per cycle), which shows an obvious improvement in the cycling performance due to the synergistic effect of CNTs and ZnS. The cycling performances of S/CNTs@ZnS with $1.27 \mathrm{mg} \mathrm{cm}^{-2}$ sulfur loading and S/CNTs with $1.3 \mathrm{mg}$ $\mathrm{cm}^{-2}$ sulfur loading at $1 \mathrm{C}$ are also shown in Fig. $5 \mathrm{a}$ and S9 respectively. The revisable discharge capacity for S/ CNTs@ZnS is 719 mAh g $^{-1}$ after 100 cycles, higher than the S/CNTs electrode. To understand the stabilities of S/CNTs@ZnS electrode, the prolonged cycling performance is evaluated at 0.5C. As shown in Fig. 5b, S/CNTs@ ZnS electrode delivers an initial capacity of $843 \mathrm{mAh}$ $\mathrm{g}^{-1}$ after activation at $0.1 \mathrm{C}$. After 500 cycles at $0.5 \mathrm{C}, \mathrm{S} /$ CNTs@ZnS electrode still retains a relative high discharge capacity of $655 \mathrm{mAh} \mathrm{g}^{-1}$. The capacity retention is calculated to be $77.6 \%$ corresponding to a capacity decay of $0.04 \%$ per cycles. Meanwhile the prolonged cycling stability of S/CNTs@ZnS at 0.5C for 1000 cycles is presented in Figure S11. During the whole cycling, the Coulombic efficiency is higher than $95 \%$. We further measured the S/CNTs@ZnS with high sulfur loading of $3.1 \mathrm{mg} \mathrm{cm}^{-2}$; the cathode shows a high discharge capacity of $670 \mathrm{mAh}$ $\mathrm{g}^{-1}$ at $0.2 \mathrm{C}$ and the capacity retention is $0.05 \%$ per cycle after 500 cycles (Fig. 5c). These results confirm that S/ CNTs@ZnS delivers outstanding electrochemical performance because of the chemical adsorption and catalytic capability of the polar ZnS to LiPSs and the improved conductivity of the CNTs@ZnS.

Finally, according to the above results and analyses, the polysulfide adsorption and conversion process on the surface of the CNTs@ZnS during discharge-charge can be illustrated schematically in Fig. 6 . The polar host material of $\mathrm{ZnS}$ can restrain the shuttle effect of polysulfides by effective chemical adsorption. Also the short chain sulfur species can rapidly result in redox on the CNTs@ZnS surface during the reaction processes due to the high electrical conductivity of CNTs and excellent chemical reaction activity of $\mathrm{ZnS}$. Meanwhile, the enhanced electrochemical performance of S/CNTs@ZnS can be attributed to the synergetic effect associated with both ZnS and CNTs. It provides higher electric conductivity for fast charge transfer of $\mathrm{Li}^{+} / \mathrm{e}^{-}$and effective 


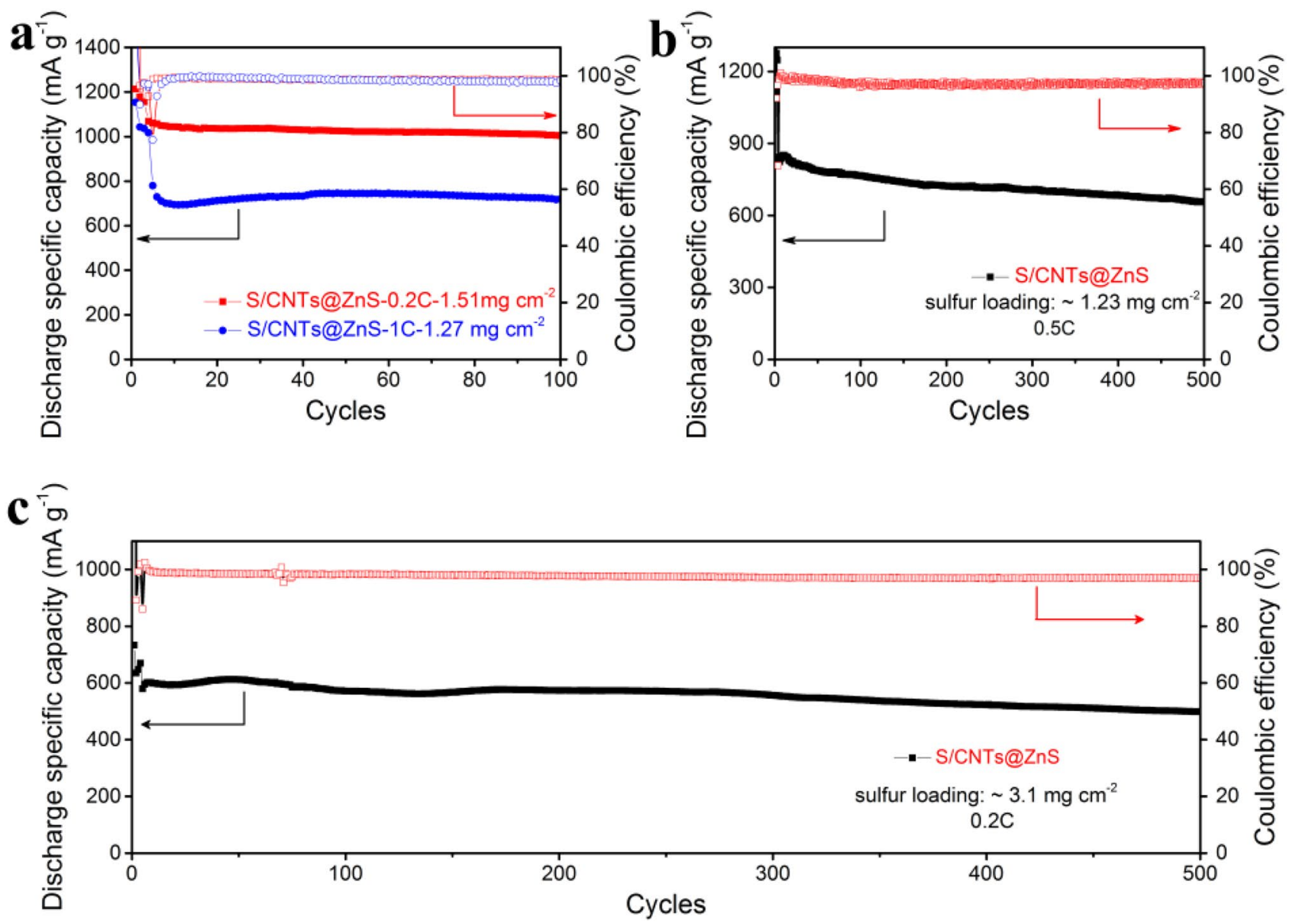

Fig. 5 Long term cycling ability of S/CNTs@ZnS. a Cycling stability at $0.2 \mathrm{C}$ and 1C of S/CNTs@ZnS; b Cycling ability at 0.5C of S/CNTs@ZnS; c Long term cycling ability of S/CNTs@ZnS at $0.2 \mathrm{C}$ with high sulfur loading of $3.1 \mathrm{mg} \mathrm{cm}^{-2}$ for 500 cycles

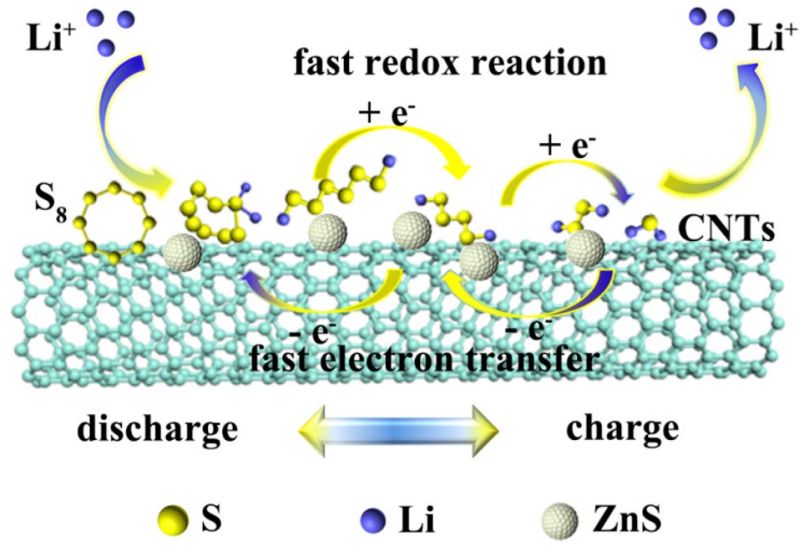

Fig. 6 Illustration of the enhanced redox reaction of the polysulfide using the catalyzing effect of CNTs@ZnS during charge-discharge

chemical adsorption of polysulfides for the conversion to $\mathrm{Li}_{2} \mathrm{~S}_{2} / \mathrm{Li}_{2} \mathrm{~S}$.

\section{Conclusions}

In summary, CNTs@ZnS composites are designed and synthesized as a host cathode material for lithium sulfur batteries using a facile wet chemical method. The host suppresses the polysulfide's shuttle effect and further enhances the observed activity. The host materials unique structure provides high conductivity for charge transfer. It also offers sufficient chemical adsorption sites to anchor the LiPSs on to the reactive interfaces of $\mathrm{ZnS}$. This in fact is responsible for the observed alleviation of the shuttle effect.

XPS reveals that the CNTs@ZnS interacts with LiPSs through the formation Li-S bonds and ZnS-S bonds. These bonds catalytically accelerate the conversion of soluble polysulfides into insoluble $\mathrm{Li}_{2} \mathrm{~S}$. The CNTs@ZnS cathode delivers an stable specific capacity with $3.1 \mathrm{mg}$ $\mathrm{cm}^{-1}$ sulfur loading after 500 cycles at $0.2 \mathrm{C}$ and ultralong cycling performance at $0.5 \mathrm{C}$ with $0.04 \%$ capacitance decay per cycle. The strategy described in this 
work provides a novel route for improving the electrochemical performance of lithium sulfur batteries.

Acknowledgements This work is supported by Key Program of the Chinese Academy of Sciences (KFZD-SW-320), China Postdoctoral Science Foundation (2019M652155) and Opened Fund of the State Key Laboratory on Integrated Optoelectronics (IOSKL2017KF08M). $M$. Yang appreciates the support from the Ningbo 3315 program. J. Wang would like to thank the financial support from the Science \& Technology Innovation Major Program of Ningbo (Ningbo 2025 Program, 2018B10056). Tiju Thomas is thankful to DST India for support via DST FILE NO. DST/TMD/SERI/HUB/1(C), and Materials for Energy Storage project.

\section{Compliance with ethical standards}

Conflict of interest There are no conflicts to declare.

\section{References}

1. Evers S, Nazar LF (2013) New approaches for high energy density lithium-sulfur battery cathodes. Acc Chem Res 46(5):1135-1143

2. Liu Y-T, Han D-D, Wang L, Li G-R, Liu S, Gao X-P (2019) NiCo2O4 Nanofibers as carbon-free sulfur immobilizer to fabricate sulfurbased composite with high volumetric capacity for lithium-sulfur battery. Adv Energy Mater 9(11):1803477

3. Liu S, Li Y, Zhang C, Chen X, Wang Z, Cui F, Yang X, Yue W (2020) Amorphous TiO2 nanofilm interface coating on mesoporous carbon as efficient sulfur host for lithium-sulfur batteries. Electrochim Acta 332:135458

4. Zhang H, Hu X, Zhang Y, Wang S, Xin F, Chen X, Yu D (2018) 3D-crosslinked tannic acid/poly(ethylene oxide) complex as a three-in-one multifunctional binder for high-sulfur-loading and high-stability cathodes in lithium-sulfur batteries. Energy Storage Mater 17:293-299

5. Ding G, Li Y, Zhang Y, Huang C, Yao X, Lin K, Shen K, Yan W, Sun F, Zhou L (2019) Waste to Wealth: exhausted Nitrogen-Doped Mesoporous Carbon/MgO Desulfurizers Turned to High-SulfurLoading Composite Cathodes for Li-S Batteries. ACS Appl Mater Inter 11(21):19096-19103

6. Peled E, Sternberg Y, Gorenshtein A, Lavi Y (1989) Lithium-sulfur battery: evaluation of dioxolane-based electrolytes. J Electro Soc 136:1621-1625

7. Manthiram A, Fu Y, Su Y-S (2013) Challenges and prospects of lithium-sulfur batteries. Acc Chem Res 46(5):1125-1134

8. Song X, Chen G, Wang S, Huang Y et al (2018) Self-assembled close-packed $\mathrm{MnO} 2$ nanoparticles anchored on a polyethylene separator for lithium-sulfur batteries. ACS Appl Mater Inter 10(31):26274-26282

9. Chen M, Zheng J, Sheng O, Jin C, Yuan H, Liu T, Liu Y, Wang Y, Nai J, Tao X (2019) Sulfur-Nitrogen co-doped porous carbon nanosheets to control the lithium growth for stable lithium metal anode. J Mater Chem A 7(31):18267-18274

10. Pope MA, Aksay IA (2015) Structural design of cathodes for Li-s batteries. Adv Energy Mater 5(16):1500124

11. Wang JL, Yang J, Wan CR, Du K, Xie JY, Xu NX (2003) Sulfur composite cathode materials for rechargeable lithium batteries. Adv Funct Mater 13(6):487-492

12. Pang Q, Liang X, Kwok CY, Nazar LF (2016) Advances in lithiumsulfur batteries based on multifunctional cathodes and electrolytes. Nat Energy 1(9):16132
13. Deng D-R, Xue F, Jia Y-J, Ye J-C, Bai C-D, Zheng M-S, Dong Q-F (2017) Co4N nanosheet assembled mesoporous sphere as a matrix for ultrahigh sulfur content lithium-sulfur batteries. ACS Nano 11(6):6031-6039

14. Ansari Y, Zhang S, Wen B, Fan F, Chiang Y-M (2019) Stabilizing $\mathrm{Li}-\mathrm{S}$ battery through multilayer encapsulation of sulfur. Adv Energy Mater 9(1):1802213

15. Xiao Z, Yang Z, Wang L, Nie H, Zhong M, Lai Q, Xu X, Zhang L, Huang $S$ (2015) A lightweight $\mathrm{TiO}_{2} /$ Graphene interlayer, applied as a highly effective polysulfide absorbent for fast Long-Life Lithium-Sulfur batteries. Adv Mater 27(18):2891-2898

16. Lang S-Y, Shi Y, Guo Y-G, Wang D, Wen R, Wan L-J (2016) Insight into the interfacial process and mechanism in lithium-sulfur batteries: an in situ AFM study. Angew Chem Inter Edition 55(51):15835-15839

17. Wei H, Rodriguez EF, Best AS, Hollenkamp AF, Chen D, Caruso RA (2017) Chemical bonding and physical trapping of sulfur in mesoporous magnéli Ti4O7Microspheres for high-performance li-s battery. Adv Energy Mater 7(4):1601616

18. Luo L, Manthiram A (2017) Rational design of high-loading sulfur cathodes with a poached-egg-shaped architecture for longcycle lithium-sulfur batteries. ACS Energy Lett 2(10):2205-2211

19. Li Z, Huang Y, Yuan L, Hao Z, Huang YJC (2015) Status and prospects in sulfur-carbon composites as cathode materials for rechargeable lithium-sulfur batteries. 92:41-63

20. Xu J, Lawson T, Fan H, Su D, Wang G (2018) Updated metal compounds (MOFs, $-\mathrm{S},-\mathrm{OH},-\mathrm{N},-\mathrm{C}$ ) used as cathode materials for lithium-sulfur batteries. Adv Energy Mater 8(10):1702607

21. Yao $Y$, Zeng $L$, Hu S, Jiang $Y$, Yuan B, Yu Y (2017) Binding $S_{0.6} \mathrm{Se}_{0.4}$ in $1 \mathrm{D}$ carbon nanofiber with $\mathrm{C}$-S bonding for high-performance flexible Li-S batteries and Na-S batteries. Small 13(19):1603513

22. Hu G, Xu C, Sun Z et al (2016) 3D graphene-foam-reducedgraphene-oxide hybrid nested hierarchical networks for highperformance Li-s batteries. Adv Mater 28(8):1603-1609

23. Wu X, Du Y, Wang P, Fan L, Cheng J, Wang M, Qiu Y, Guan B, Wu H, Zhang N, Sun K (2017) Kinetics enhancement of lithium-sulfur batteries by interlinked hollow $\mathrm{MoO} 2$ sphere/nitrogen-doped graphene composite. J Mater Chem A 5(48):25187-25192

24. Chen A, Liu W, Hu H, Chen T, Ling B, Liu K (2018) Three-dimensional TiO2-B nanotubes/carbon nanotubes intertwined network as sulfur hosts for high performance lithium - sulfur batteries. J Power Sources 400:23-30

25. Wei Seh Z, Li W, Cha JJ, Zheng G, Yang Y, McDowell MT, Hsu PC, Cui Y (2013) Sulphur-TiO2 yolk-shell nanoarchitecture with internal void space for long-cycle lithium-sulphur batteries. Nat Commun 4:1331

26. Li X, Lu Y, Hou Z, Zhang W, Zhu Y, Qian Y, Liang J, Qian Y (2016) SnS2-Compared to SnO2-Stabilized S/C Composites toward High-Performance Lithium Sulfur Batteries. ACS Appl Mater Inter 8(30):19550-19557

27. Li Z, Zhang J, Lou XW (2015) Hollow carbon nanofibers filled with $\mathrm{MnO} 2$ nanosheets as efficient sulfur hosts for lithium-sulfur batteries. Angew Chem Inter Edition 54(44):12886-12890

28. Ni L, Wu Z, Zhao G, Sun C, Zhou C, Gong X, Diao G (2017) Coreshell structure and interaction mechanism of gamma- $\mathrm{MnO}_{2}$ coated sulfur for improved lithium-sulfur batteries. Small 13(14):1603466

29. Lin H, Zhang S, Zhang T, Cao S, Ye H, Yao Q, Zheng GW, Lee JY (2019) A cathode-integrated sulfur-deficient Co9S8 catalytic interlayer for the reutilization of "lost" polysulfides in lithiumsulfur batteries. ACS Nano 13(6):7073-7082

30. Jiang $S$, Chen $M$, Wang $X, W u Z$, Zeng $P$, Huang $C$, Wang $Y$ (2018) MoS2-coated N-doped mesoporous carbon spherical composite cathode and CNT/Chitosan modified separator for advanced lithium sulfur batteries. ACS Sustain Chem Eng 6(12):16828-16837 
31. Fang R, Zhao S, Sun Z, Wang DW, Cheng HM, Li F (2017) More reliable lithium-sulfur batteries: status, solutions and prospects. Adv Mater 29(48):1606823

32. Huang SZ, Lim YV, Zhang XM, Wang Y, Zheng Y, Kong DZ, Ding M, Yang SYA, Yang HY (2018) Regulating the polysulfide redox conversion by iron phosphide nanocrystals for high-rate and ultrastable lithium-sulfur battery. Nano Energy 51:340-348

33. Li G, Sun J, Hou W, Jiang S, Huang Y, Geng J (2016) Three-dimensional porous carbon composites containing high sulfur nanoparticle content for high-performance lithium-sulfur batteries. Nat Commun 7:10601

34. Su D, Cortie M, Fan H, Wang G (2017) Prussian Blue Nanocubes with an Open Framework Structure Coated with PEDOT as High-Capacity Cathodes for Lithium-Sulfur Batteries. Adv Mater 29(48):1700587

35. Fu K, Gong Y, Hitz GT, McOwen DW et al (2017) Three-dimensional bilayer garnet solid electrolyte based high energy density lithium metal-sulfur batteries. Energy Environ Sci 10(7):1568-1575
36. Wang Y, Zhang R, Pang Y-c, Chen X et al (2019) Carbon@titanium nitride dual shell nanospheres as multi-functional hosts for lithium sulfur batteries. Energy Storage Mater 16:228-235

37. Wang $C$, Wan W, Chen J-T, Zhou H-H, Zhang X-X, Yuan L-X, Huang Y-H (2013) Dual core-shell structured sulfur cathode composite synthesized by a one-pot route for lithium sulfur batteries. J Mater Chem A 1(5):1716-1723

38. Yun JH, Kim J-H, Kim DK, Lee H-W (2018) Suppressing polysulfide dissolution via cohesive forces by interwoven carbon nanofibers for high-areal-capacity lithium-sulfur batteries. Nano Lett 18(1):475-481

Publisher's Note Springer Nature remains neutral with regard to jurisdictional claims in published maps and institutional affiliations. 